\title{
Socially assistive robots: current status and future prospects for autism interventions
}

This article was published in the following Dove Press journal: Innovation and Entrepreneurship in Health

\author{
Laurie A Dickstein-Fischer' \\ Darlene E Crone-Todd ${ }^{2}$ \\ Ian M Chapman' \\ Ayesha T Fathima ${ }^{3}$ \\ Gregory S Fischer ${ }^{3}$ \\ 'Secondary and Higher Education \\ Department, School of Education, \\ Salem State University, Salem, MA, \\ USA; ${ }^{2}$ Psychology Department, \\ College of Arts and Sciences, Salem \\ State University, Salem, MA, USA; \\ ${ }^{3}$ Robotics Engineering Program, \\ Worcester Polytechnic Institute, \\ Worcester, MA, USA
}

\begin{abstract}
Social robots interact with people through social interactions, physical assistance, and therapy delivery. Socially assistive robots (SARs) are specifically intended to assist in a therapeutic way to help build social behavior skills. One area where SARs have gained significant attention is in the treatment and diagnosis of autism spectrum disorder. With the increase in rates of individuals diagnosed with autism spectrum disorder, there is an increasing need for equitable and accessible applied behavior analysis (ABA) therapy services. Current barriers for sufficient access to therapy include high administrative burden, burnout rates on the part of ABA therapists, and affordability of services. One way to reduce administrative burden is to automate procedures using computer-based interventions, including SARs. SARs have the potential to increase client engagement, while at the same time making it possible for therapists to provide a more interactive session for their client. We argue that there is benefit to clients, therapists, scientists, and to the general public if behavior analysts can work with robotic design engineers for further research and development on SARs. Such collaboration is necessary for ensuring that robust SAR designs are embedded in conceptually systematic approaches to ABA, while at the same time integrating relevant engineering design considerations. This technological integration is important for the field of ABA to move forward as robotics become more commonplace, and to effectively address the cost, quality, and access considerations involved.
\end{abstract}

Keywords: SARs, applied behavior analysis, autism therapy, therapeutic robots, human robot interaction, PABI, psychology, technology, computer aided instruction, ASD

\section{Introduction}

During the past decade, a number of social robots with the ability to autonomously interact have been investigated. ${ }^{1-3}$ There are a number of ways in which a social robot can interact with a person, with them typically classified as "socially interactive", "contact assistive", or "socially assistive" robots. ${ }^{4}$ The use of technology to mechanize some aspects of therapy has been studied in various settings and can serve as a way to enable greater access to needed services, improved and more consistent outcomes, and enhanced efficiency in therapy delivery. ${ }^{5-7}$ Socially assistive robots (SARs) can assist therapists and caregivers in the teaching of skills in the form of relatively lower cost portable systems that can potentially combine the use of virtual reality, computer-aided instruction, artificial intelligence, and video interventions due to their modularity as a feedback-driven programmable system. In the past decade, the use of SARs has gained attention in the treatment and diagnosis of individuals with autism, ${ }^{8-10}$ and research focusing on SAR technologies for autism treatment has commonly presented an increase in user engagement, enhanced user attention levels, and improvement in social skills. ${ }^{9}$
Correspondence: Laurie A

Dickstein-Fischer

School of Education, Salem State

University, 352 Lafayette St. SB-300-A,

Salem, MA 01970, USA

Tel +l 9785422789

Email Idicksteinfisc@salemstate.edu
Innovation and Entrepreneurship in Health 2018:5 I5-25 
A number of studies suggest that children with autism can be more comfortable interacting with robots, possibly because the robots' behavior and reactions are more consistent and predictable than that of humans. ${ }^{11-15}$

As the prevalence of individuals diagnosed with autism spectrum disorder (ASD) is at a historically high rate of 1 in 68 children, ${ }^{16}$ there is a concomitant need for access to services. The Diagnostic and Statistical Manual of Mental Disorders, 5th edition (DSM-5) characterizes autism as a "persistent deficits in social communication and social interactions across multiple contexts" (p. 50). ${ }^{17}$ As autism is a spectrum disorder, it presents at varying levels; the DSM-5 categorizes those diagnosed with ASD into three levels "requiring very substantial support", "requiring substantial support", and "requiring support" - but even within those designations there are varying levels of symptomology and ability. ${ }^{17}$ Additionally, autism is diagnosed more in males than in females with a ratio of $4: 1 .{ }^{17-20}$ To explain this, Skuse suggests that the gene for autism is located on the $\mathrm{X}$ chromosome. ${ }^{19}$ Females inherit an X chromosome from both parents, and males inherit one. The X chromosome which girls inherit from their fathers contains an imprinted gene which "protects" the carrier from autism, thus making girls less likely to develop the condition than boys. ${ }^{19,21}$

Applied behavior analysis (ABA; formally known as the Lovaas Model) is one of the most widely used interventions for the treatment of ASD and has been proven to be effective. ${ }^{22}$ ABA "has the distinction of having the longest history and the most extensively documented evidence base to support its efficacy in the treatment of autism" (p. 13). ${ }^{23}$ One important technique an ABA therapist uses is discrete trial training (DTT) in which tasks are broken down and appropriate behavior is reinforced. The tasks are sequenced and prompts are presented strategically along with measurement and analysis of the performance as the therapy progresses. Through strategic teaching and repeated trials, ABA has proved to be developmentally advantageous for children with autism. ${ }^{24-26}$

The prevalence of ABA therapies and interventions has been increasing; however, despite its demonstrated effectiveness with respect to individuals on the spectrum, access to ABA is not equal. In this article, we argue that the combined use of ABA with technology in the form of socially assisted robots is one important way to overcome some of the barriers to obtaining effective ABA interventions. These barriers include affordability, administrative burdens on therapists, and high burnout rates. To overcome these barriers, SARs can be designed to embed ABA technology in terms of physical and programmatic considerations, so long as there is also a conceptually systematic way in which the design is developed and carried out. ${ }^{27,28}$ We believe it will benefit clients, therapists, scientists, and the greater public if both therapists and experimental behavior analysts can work with robotics design engineers to conduct the types of research and development on SARs that can lead to a conceptually systematic synthesis and evaluation of robotics and behavior analysis. Doing so could usher in an era in which such robots would be available to effectively assist applied work for the benefit of both health care personnel and clients/students.

\section{Making the case for SARs in ABA therapy: accessibility, affordability, and administrative burden}

In the United States, the mean lifetime cost of supporting an individual with an ASD and intellectual disability has been found to be \$2.4 million USD, and \$1.4 million USD for an individual with an ASD without intellectual disability, with one of the main costs being special education services. ${ }^{29}$ About a decade ago, the average annual cost for intensive one-on-one ABA services ranged from $\$ 33,000$ to $\$ 60,000$ USD, ${ }^{30,31}$ with estimates now between $\$ 46,000$ and $\$ 47,500$ USD per year. ${ }^{32}$ Depending on the severity of the diagnosis, age of the child, and intensity of intervention required, these costs could be even higher. Behavior therapists as a group also experience occupational burnout and procedural drift as a result of the intensive nature of the multitasking involved in data collection procedures during long hours of intense one-to-one personalized sessions. ${ }^{33-36}$ Furthermore, there is concern among all key players (e.g., parents/caregivers, clients, supervisors, and behavior analysts) regarding not only the relatively high turnover, but also the unreliability, of some therapists in the field. ${ }^{37}$ Hurt et al point out that when there are changes in the individuals providing ABA therapy, the children with ASD lose precious time and consistent progress. ${ }^{38}$ As in any helping profession in which there is high turnover, occupational burnout, and procedural drift combined with a high cost of conducting therapy, there is often a need to automate tasks and subtasks within ABA sessions, analyses, and reporting.

The use of technology automating some aspects of therapy has been studied in various settings; the technologies studied include, but are not limited to, virtual reality, computer-aided instruction, DVD- or video-based interactions, and robotics. ${ }^{5-7}$ For a comprehensive treatment such as ABA to target the acquisition of multiple social or other behavioral skills, we propose that the use of robotics, specifically social robots, can provide a comprehensive approach for dissemination of the therapy. Feil-Seifer and Matarić differentiate 
social robots from "contact assistive robots", which assists physically disabled humans in their mobility. Instead, they categorize social robots as either "socially interactive robots" that are mainly used as a form of entertainment for humans of all ages and abilities, or "socially assistive robots" that assist humans with social behavior skills. ${ }^{4}$ Social robots can assist therapists and caregivers in the teaching of skills in the form of relatively lower cost portable systems that can potentially combine the use of virtual reality, computer-aided instruction, and video interventions due to their modularity as a feedback-driven programmable system.

In the past decade the use of SARs has gained attention in the treatment and diagnosis of individuals with autism, ${ }^{8-10}$ and research focusing on SAR technologies for autism treatment has commonly presented an increase in user engagement, enhanced user attention levels, and improvement in social skills. ${ }^{9}$ However, Kim et al described how clinicians have been hesitant thus far to adopt robotic technologies because of a lack of a clear case for their utility; they further note that clinical research must be driven by specific needs of the target population and not by capabilities of the robots. ${ }^{39}$ Begum et al presented a recent review paper with the primary focus of understanding how current robot-mediated interventions stand with respect to evidence-based practice. ${ }^{10}$ The authors noted that in their survey of studies to date, all human-robot interaction (HRI) studies performed with individuals with ASD demonstrated elevated enthusiasm, increased attention, imitation ability, utterances, and social activity when interacting with the robot. They further describe neurobiological evidence including functional magnetic resonance imaging demonstrating that a robot may be perceived as a social interaction partner in a way similar to a human, and that robotic movements elicit visuomotor priming. ${ }^{10}$

A number of studies suggest that autistic children can be more comfortable interacting with robots, possibly because the robots' behavior and reactions are more consistent and predictable than that of humans. ${ }^{11-15,40}$ Children with autism often have trouble reading and responding in a socially appropriate way to other people's emotional expressions; however, with SARs the child may be more readily engaged because of the predictability of the interactions. In a study conducted by Scassellati, he found that children with autism responded to a basic robot in the same manner as their typically developing peers, including smiling, making eye contact with the robot, and vocalizing. In fact, while typically developing children sustained interaction with the robot only when it was responsive, the children with autism sustained interaction whether the robot was responsive or not. ${ }^{12}$ In a review of the clinical use of robots for autism therapy, Diehl et al described in regard to responses to robots, individuals with ASD prefer interactive robots over passive objects, initially prefer robot-like characteristics in social interaction, and respond faster to robotic movement cues. ${ }^{15}$

The use of robotic technology may further the opportunity for individuals diagnosed with ASD to mediate a social behavior. ${ }^{40}$ Dautenhahn and Werry hypothesized that by slowly increasing the unpredictability of a robot's interactions, children with autism could be guided toward more complex interactions that might be found in social human to human interaction. ${ }^{40}$ Thus, an important first step in easing the accessibility and affordability of therapy to children with ASD is to incorporate SARs that aid in the dissemination of "empirically supported treatments such as applied behavior analysis in order to examine whether there are specific benefits beyond general therapeutic change to including such an agent in therapy" (p. 258). ${ }^{15}$ Efficacy trials of this nature are needed at this early stage of SAR development in order to ensure that they meet the standards of evidence-based practice for ABA treatment.

However, as noted by Diehl et al, there are limited studies that have shown clinical applications of a robot with individuals with ASD, and of those very provided statistically significant results, and there is a paucity of literature in ASD-related journals. ${ }^{15}$ Thus, as described by Begum et al, it is essential that SARs being developed based on the above-noted potential benefits be tested according to rigorous standards to ensure evidence-based support for their use in clinical practice. ${ }^{10}$

Another potential benefit of SARs is that their application may help to lower overall costs associated with ABA therapy, which would therefore increase this treatment's accessibility for lower income families. Many SARs are either in a developmental stage or are solely for research purposes, and therefore, have no determined sales price, but a few are available to consumers. For example, the Nao robot is $\$ 7,990$ USD and is paired with educational materials that are $\$ 4,200$ USD per year, ${ }^{41,42}$ and another robot, Milo, costs \$5,000 USD, which includes full curriculum. ${ }^{43}$ This, compared with the estimated cost of between $\$ 46,000$ and $\$ 47,500$ USD per year for ABA services, ${ }^{32}$ is a significant reduction.

SARs can not only provide much needed social assistance to children diagnosed with ASD, but also manage the administrative responsibilities in conducting therapy as a programmable system that can address the different needs of children with ASD. These systems can be used to administer sessions, collect data, analyze interactions, and generate reports and graphs. Thus, they are potentially a powerful asset to any ABA 
therapist, their student(s) or client(s), and others involved in the progress of the individual. While the main practical advantages of using such robots for effective interventions are their potential for accessibility, easier administration, and lower costs, it has also been suggested that some SARs can also act like an interactive toy playmate for children with autism. ${ }^{8,44}$ If so, then the SAR could potentially provide additional conditioned reinforcement in between sessions. Welch et al noted that robotic technology allows for consistency in terms of stimuli being presented, and in quantifying and recording performance. ${ }^{44}$ Such standardized monitoring is used to facilitate diagnosis, intervention assessments, and so forth may reduce administrative burden on the part of therapists. In doing so, more efficient time can be spent during sessions, which should lead to greater gains in behavior. Of course, this would not only suggest that the technology be a high quality synthesis of behavior analysis and robots, but also that it is designed in a manner that increases engagement.

\section{Increasing engagement and visual appeal consideration}

A number of social robots with the ability to autonomously interact have been investigated, especially as assistive tools for engaging autistic children. ${ }^{1-3}$ According to Robins et al, therapeutic playmates help to improve communication skills, and introducing a robotic toy can help to mediate the skill acquisition process by encouraging children with autism to explore a range of play styles. ${ }^{13}$ SARs have also been shown to improve eye contact in children with $\mathrm{ASD},{ }^{14}$ as well as promote social behaviors such as social and communicative interaction, eye contact, and other socially important behaviors. ${ }^{45}$ Tariq et al also found that the social interactions seamlessly generalized from robot-child to child-human interactions. ${ }^{45}$ Thus, SARs could be used as an effective tool to increase engagement on the part of children with ASD while conducting empirically based ABA treatments.

Cabibihan et al identified and classified the design of such robots "according to appearance, functionality, safety requirements, autonomy, modularity and adaptability" (p. 596). ${ }^{3}$ These robots seek a visual appeal that is engaging in therapy by using bright colors, but not so bright that it distracts the child. Robots with rotating mechanical parts, different shapes, and the use of lights are also considered appealing, 3,46 possibly due to the novelty of the stimuli involved. However, a balance is desired such that the robot is "not so mechanical that the child gets more interested in examining the robot's mechanical parts" (p. 596) than in the task(s) at hand. ${ }^{3}$ Some social robots that are presently in use in the market that match these criteria include the following: 1) Keepon with its bright yellow color and round shape, 2) Paro with its seal shape and plain white or other colors, 3) Popchilla with its bright blue or green color and antennas, and 4) Pleo with its dinosaur shape and green color. ${ }^{47-49}$

Some children tend to show decreases in social engagement behaviors if the robots too closely resemble humans. ${ }^{3,11,13}$ In a study conducted by Robins et al, researchers compared the reactions of children with autism when interacting with two types of humanoid robots: one that appeared more human-like and one with plain features. Their results indicated that the children had a preference for the featureless robot over the human-like robot. ${ }^{13}$ At present, it is uncertain whether this preference for characteristics that are less humanoid is developmental, or if it is consistent across the lifespan, as evidenced by the "Uncanny Valley" phenomenon in which the more human-like a mechanical or robotic figure is, the more discomfort humans self-report toward that figure. ${ }^{50,51}$ Therefore, the "realism" aspect of the appearance seems to play an important role as some children with autism show more engagement with robots that look less like a human. ${ }^{3}$ The abovementioned robots actually abide by this finding and have a pet-like appearance with modular features. Moreover, evidence for the Uncanny Valley phenomenon comes from both experiments and surveys which indicate children's preference for non-anthropomorphic and biomimetic robots. ${ }^{3,13}$ These features have also been incorporated into the social robots discussed above. Despite the uneasiness typically felt by children with ASD around realistic humanoid robots, some anthropomorphic robots for conducting autism therapy are also being researched, such as Zeno R-50, Nao, and KASPAR. ${ }^{40,52,53}$ Ricks and Colton pointed out that the comparatively less realistic and less complex appearance of, for instance, KASPAR, helped the children with ASD to more often attend to KASPARs face. ${ }^{14}$ In addition, the children were less likely to display behaviors often labeled as "anxious" or "overloaded", which they had a history of doing around humans. Similarly, the recommended size of the robot is roughly determined to be appropriate to the size of the child for ease of making eye contact and to be less intimidating in general. ${ }^{3}$ The aforementioned social robots (e.g., Nao, Kaspar, Zeno R-50, Keepon, Popchilla, Pleo, and Paro) all abide by the recommended appropriate size for these children as the former humanoids are roughly the same size as the child. However, the latter nonhumanoids are even smaller, and can fit like a toy in the child's hands.

While these physical appearance characteristics and their relationship to increased likelihood of attending to the SAR 
are necessary considerations, they are not sufficient to be effective in assisting with ABA therapy. What is needed in addition to these considerations is a robust design that is also embedded in behavior analysis. We now turn to an overview of the considerations that are needed regarding the environmental and conceptually systematic aspects.

\section{Creating robust SAR designs embedded in behavior analysis}

When creating a socially assistive robot, there are initial considerations regarding the durability of the robot, safety features, and the modularity and adaptivity of the programming. These are the physical and logical aspects, then, that go into the development of an SAR. In terms of use in ABA settings, however, there is a need for a conceptually systematic understanding of how and why certain aspects of the SAR either do or do not work effectively to carry out, or assist in, the delivery of therapy. Given these needs and potential advantages, various ways of designing such robots are also being explored to provide the best possible experience and effectiveness in therapeutic application of robotics. Doing so in a manner consistent with behavior analysis would be a way to realize Skinner's vision of using technology to provide better educational outcomes for individuals. ${ }^{54}$ For example, Skinner's use of teaching machines in the past century helped usher in direct instruction, Keller's personalized system of instruction (PSI) ${ }^{55}$ Pear's computer-aided PSI, ${ }^{56}$ and so many other adaptations involve using machines or computers to automate much of the work involved in carrying out robust teaching strategies.

\section{Robust design: interaction, safety, autonomy, modularity, and adaptability considerations}

\section{Interactions with the user}

When considering SARs one must understand not only the physical robot design, but also the design in terms of HRI, and the method of evaluating HRIs. ${ }^{9}$ In regard to the physical design, social robots have taken many different forms, ranging from simple to complex types of varieties of anthropomorphism approximations (e.g., human-like, animalistic, or mechanical, as mentioned previously) in their appearance. ${ }^{9}$

\section{Safety and robustness of the physical embodiment}

Safety requirements, autonomy, and modularity/adaptability of any robot are must-have design requirements, especially with SARs used for therapeutic purposes. Cabibihan et al suggest that there are several considerations for a robust design that is needed to ensure sturdiness and safety. ${ }^{3}$ These include the risk of the robot being thrown on the ground or against the wall, a lack of sharp edges, ensuring that the movements are not too fast or jerky, and minimizing the likelihood of robotic malfunctions.

\section{Autonomy, modularity, and adaptability of the software}

Cabibihan et al pointed out that semiautonomous robots are needed to avoid the therapist from being ever-present to perform every action of the robot, and to allow the therapist to choose best actions in response to the child's behavior when needed. ${ }^{3}$ Cabibihan et al also pointed out the importance of modularity and adaptability in design of the robotic system. ${ }^{3}$ According to Cabibihan, modularity would allow for children to choose features that can sustain their interest and would reduce repair costs. ${ }^{3}$ Modularity of the robot is also necessary to allow for changes in the functionality of the robot, whether it is with different children or with different abilities of these children. Adaptability in the robotic design is also an important consideration not only for the environment in which the child-robot interaction takes place, but also with respect to the potential for the SAR to respond appropriately to progressively more complex development on the part of the child. ${ }^{3}$

Hence, all these design factors need to be taken into consideration for the socially assistive robot to adhere to the therapy standards accordingly. Of course, all of these considerations must be made in combination within a conceptually systematic framework embedded in behavior analysis. At a minimum, a consideration of the SAR actions in terms of stimulus characteristics, preference assessments, and potential for providing consequences to change behavior in the desired direction should all be part of the considerations in design.

\section{Possible SAR actions, stimulus characteristics, preference/choice, and consequences}

In addition to the functions described earlier, Cabibihan et al further divided the functionality of the robot into "sensory rewards", "locomotion", and "choice and control". Sensory stimuli in the form of "lighting up of the robot's body part, or the playing of music, or the robot's clapping" (p. 597) are considered intriguing to some children with autism because they tend to allocate more time paying attention to the robot when it is emitting these types of stimuli. ${ }^{3}$ Thus, 
the sensory stimuli may serve as antecedent or consequential stimuli; however, the ability of the stimuli to serve in these capacities is an empirical question for each situation and the individuals involved. As an example, Paro, the seal-shaped robot, has tactile sensors that can react to human touch. ${ }^{49}$ The humanoid robots such as Nao and Zeno R-50 are capable of clapping their hands while Nao also has the ability to light up with its LED lights in response to any type of contact. ${ }^{53}$ The nonhumanoid Pleo robot is capable of playing music while dancing. ${ }^{47}$ The sensory stimuli can thus potentially act as discriminative stimuli, or conditioned reinforcers, during sessions. If so, these actions on the part of the robot can be effectively incorporated into ABA interventions.

Since sensory stimuli may not serve as reinforcers for all children with ASD, personalized use of reinforcers is considered a plus for a successful intervention, which is determined using preference assessments and verified using appropriate reinforcer assessments. ${ }^{57}$ Skilled therapists conduct the therapy while leveraging the individual motivating operations for the child at hand, ${ }^{58,59}$ thereby increasing the probability that the child will be more likely to engage in activities of their choice, ${ }^{60}$ and use effective reinforcers. For instance, the Keepon social robot leverages any positive social interactions made by the child by consequently rocking and bouncing from side to side as a show of excitement in the form of a consequence when the child increases interactive behaviors. ${ }^{14}$ Research has demonstrated that robots with mechanical moving parts lead to more interactions during play, when compared with static or passive toys. ${ }^{61}$ For example, social robots like Pleo and Keepon use dance movements as a form of therapy for children with ASD. ${ }^{40,48}$ Giving a child with autism the control to choose these aspects of the robot, such as types of consequences for appropriate behavior, will lead to a higher probability of engagement. ${ }^{3,13}$ However, when designing a robot, it is important to make it modular, adaptable, and therefore, customizable for children with ASD who may not respond well to "sensory rewards".

With the physical and conceptual considerations now laid out, we turn to a discussion of the current SARs' capabilities, and how future developments may emerge in this exciting field.

\section{Current SARs' capabilities and future developments}

A number of the aforementioned SARs are in the market or undergoing research and development for their utility. In a review of research related to robot-mediated ASD interventions, Begum et al suggest that researchers designing robots for these types of interventions should consider the following four general summary categories as intervention goals: social, communication, maladaptive behavior, and academic skills. ${ }^{10}$ Many of the off-the-shelf social robots that are currently being studied as tools for autism therapy target only one of these skill categories. Further, few robots included in SAR meta-analyses rely on any specific empirically supported therapy methodology, such as ABA, and these few exclusively focused on a single skill development. ${ }^{7,10}$ The humanoid robots such as Zeno R-50, Nao and Kaspar, or nonhumanoid robots such as Pleo, Paro, Keepon, or Popchilla are being studied as vehicles for exclusively promoting social skills for children with autism. Among these robots, the humanoid Zeno R-50 was specifically used by Salvador et al to incorporate the ABA therapy approach but, once again, only focused on socioemotional skill development. ${ }^{53}$ Similarly, the Nao robot also utilized ABA-based intervention in the social skills domain. ${ }^{10}$ For the behavior analyst, these constructs would need to be further operationally defined in consultation with the designer to create appropriate, adaptive modules that can be presented in collaboration between the SAR, the therapist, and the child.

A relatively new SAR, the Penguin for Autism Behavioral Intervention (PABI), is currently being developed from the ground up as a modular platform specifically designed for assisting in the delivery of ABA therapy. ${ }^{62}$ PABI has the ability to provide programming related to various sets of skills, including the goals of intervention mentioned by Begum et al, ${ }^{10}$ using several of the features of empirically supported ABA therapy, such as DTT. As research and development continue, other modules utilizing other types of intervention can be added or modified. PABI is a non-anthropomorphic small-sized, accessible social robot that is designed for effective early interventions (EIs) in children with autism (Figure 1). ${ }^{62}$ As an SAR built upon the previously discussed concepts of modularity and adaptability, PABI is intended to evolve as feasibility studies are conducted for its clinical applications and behavioral underpinnings..$^{8,62,63}$ The program utilizes a flashcard database to provide small units of mastery-based criteria for each session, and can incorporate cloud hosting by storing its flashcard database on the cloud for ease of accessibility and curriculum management. These flashcards can utilize multimedia, including images, text, video or audio clips, which can then be modularly selected for creating new programs such as DTTs for ABA therapy (Figure 2). As therapy continues, the modules could, for 


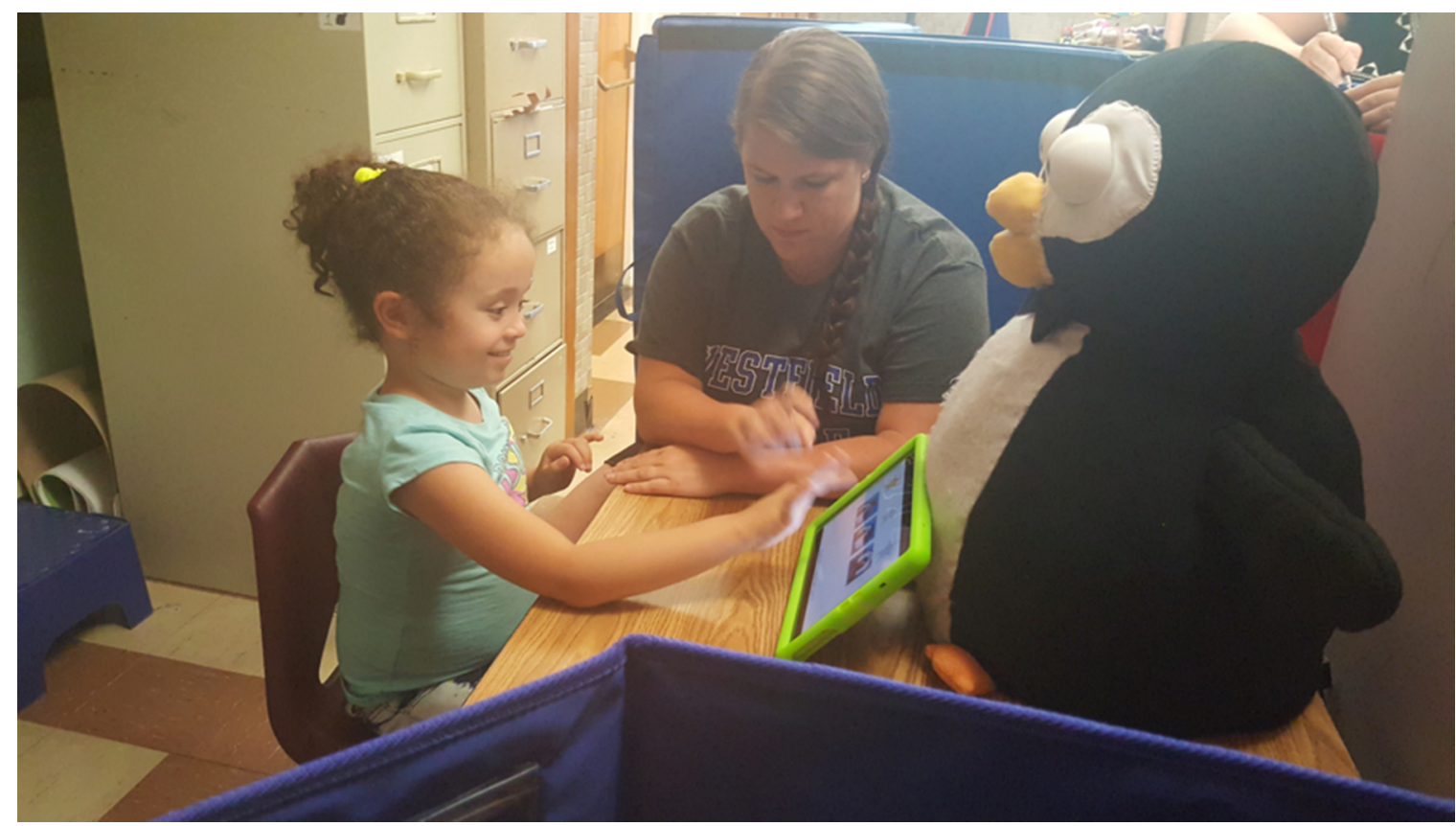

Figure I The PABI robot in a triadic therapy scenario providing DTT ABA therapy for an autistic child in conjunction with a therapist.

Note: Copyright @2017. Adapted from Dickstein-Fischer LA, Pereira RH, Gandomi KY, Fathima AT, Fischer GS. Interactive tracking for robot-assisted autism therapy. In: Proceedings of the Companion of the 2017 ACM/IEEE International Conference on Human-Robot Interaction; March 20I7; I07-I08; Vienna, Austria. ${ }^{63}$

Abbreviations: ABA, applied behavior analysis; DTT, discrete trial training; PABI, Penguin for Autism Behavioral Intervention.

example, eventually be programmed to change over to free operant procedures, and incorporate progressive schedules of reinforcement. When conducting therapy, sensory stimuli can be provided with PABI's physical features, such as wing flapping or face tracking. The language used by PABI can also be extensible, thereby catering to children with diverse backgrounds and spoken languages, as a multilingual robot with English, Spanish, and many other languages being easily programmed as the language of instruction.

Since ABA therapists have a limited number of hours of availability in a given time period (e.g., per day or per week), developers of ABA-related SARs hope to fill time gaps in treatment by creating robots that can be used either autonomously or remotely to assist therapy. As an example, PABI is being developed to be used autonomously to assist ABA therapy anywhere and at any given time of the day, thereby with the potential to provide more accessibility and free up time and resources on the part of therapists. PABI can also provide continuous assessment with future applications in distance learning, so that a therapist and/or a supervisor can $\log$ in remotely for conducting therapies, providing distance supervision, or accessing the behavior assessment results database. This feature of remotely controlling the robot's movements outside of the view of the users who speak to it (i.e., the "Wizard of Oz" technique) has been used in studies with the Pleo robot, wherein the reliability of the robot was solicited using the Wizard of $\mathrm{Oz}$ technique to control the robot's movement in response to speech. ${ }^{39}$ However, as pointed out by Scassellati et al, such techniques are effective only for prototyping and not for large-scale use. ${ }^{9}$ Hence, PABI's current capability to be controlled remotely is considered only as a means to acquire and measure data in certain circumstances so as to develop a more adaptable system, and not as a primary method.

As mentioned earlier, another important feature employed by SARs is eye gaze tracking. For example, robots like Keepon, Kaspar, and PABI use gaze tracking which could be used for either diagnosis or for the purpose of increasing socially desirable behavior. Detecting gaze direction is indicative of early vulnerability for autism in infants and toddlers, ${ }^{12}$ and thus SARs can be highly valuable tools for therapists for detection of those who require EI for achieving the highest attainable progress. ${ }^{64}$ Post-processing of eye gaze estimation, along with face recognition and head pose estimation, has been carried out in pilot studies with PABI. ${ }^{63}$ As such, future development of PABI can involve leveraging its live face and gaze tracking while conducting the therapy, to observe the subsequent effects of the therapy on socially desirable behaviors, such as sustaining eye contact over a given amount of time, and whether or not prompting was 


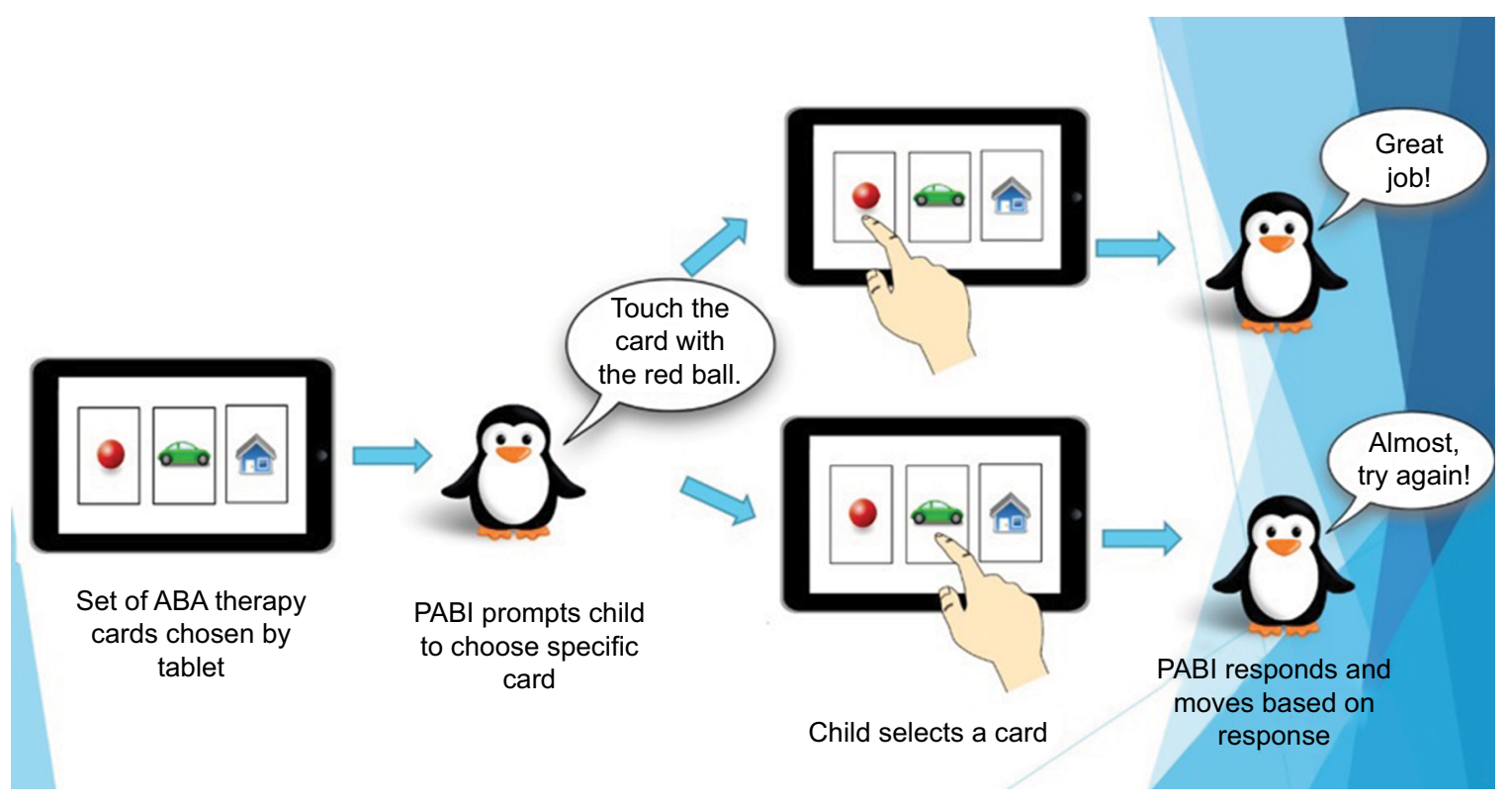

Figure 2 A flowchart showing how a socially assistive robot can assist in discrete trial training.

Note: The PABI interacts with a child by autonomously providing instruction, rewarding or correcting, and reacting with sensory stimuli.

Abbreviations: ABA, applied behavior analysis; PABI, Penguin for Autism Behavioral Intervention.

required. Based on the experimental setup, it is also possible to develop SARs like PABI so that a report can be generated for functional behavior analyses. In these cases, PABI's behavior/gesture/emotion recognition technology is currently being developed to identify patterns of behavior displayed by the individuals at any given point of time during therapy, and in relation to the antecedent and consequential events during the session. These reports can help to ascertain and mitigate the controlling variables involved in problem behaviors with post-processing of the data, the results of which can lead to functional hypotheses regarding intervention plans. Studies of differential reinforcement of alternative behavior indicate that treatment integrity failures leading to reinforcement of problem behavior is more detrimental than failing to reinforce appropriate behavior. ${ }^{65}$ Thus, having a video recording report analysis via SARs such as PABI could lead to fewer treatment integrity failures. Moreover, using SARs technology increases the likelihood of procedural integrity with respect to implementation of interventions while reducing the opportunity for human error. ${ }^{6}$

Some of the work that is conducted during the intervention, such as work on social skills or communication, may be facilitated with the use of the robot. The SAR may help by requiring less time from highly trained professionals (i.e., an individual who has completed training through the master's or doctoral level), and providing more assistance to front-line therapists or care givers who are under the supervision of the more highly trained professional. As an example,
PABI's face tracking capabilities can be used as a modeling strategy during ABA sessions, as it models eye-to-eye gazing. Overall, PABI's modular technology and programming can be extended through research and development to implement various other fully functional ABA intervention therapy applications. That is not to say a robot will replace humans in the intervention, but if the social robot leads to the child spending more time on the task at hand, then there should be greater gain in skills, rather than time spent redirecting a child who may be described as unruly, inattentive, or tantruming. This advantage of such triadic interaction (i.e., between the SAR, therapist, and child) is also reaffirmed by Reed et al when they noted that using technology "allows practitioners to engage in other work-related tasks or simultaneously work with more individuals at one time" (p. 1004). ${ }^{6}$ Another advantage of such a triadic interaction is the generalization of skills beyond the child-robot interaction to child-human interaction. As Ricks and Colton noted, increased social interactions between the experimenter/therapist and the child with autism were evoked by the robot through the triadic interaction. ${ }^{14}$ Following this triadic interaction, a dyadic interaction can be gradually introduced by fading the role of the robot and programming for the generalization of social skills specifically as proposed in the meta-analyses by Begum et al. ${ }^{10}$ It is also likely that the role of the therapist can be adjusted to include more interactive and assistive behaviors during sessions, since the manual record-keeping and stimuli presentation demands would be reduced. This could include the benefit of providing families 
with the opportunity to continue treatment when using SARs like PABI if unexpected or planned events require their child to spend time away from his or her therapist. With a portable SAR, parents could also be trained to carry out some of the programming, with the additional benefit of continuing distance observation by the therapist or supervisor.

Overall, social robots can provide children diagnosed with ASD with an intervention experience that increases their attention, time-on-task, and can utilize behavioral approaches to motivation and engagement in therapy. ${ }^{12,48}$ At the same time, social robots allow for more flexibility for the clients served, their parents and caregivers, and the therapists.

\section{Conclusion}

Technological interventions (such as social robots) may be an affordable and effective option that should be incorporated to increase access and affordability and consistency of ABA services, and to reduce the administrative burden on therapists. Progress in children with autism is affected by accuracy, consistency, reciprocity, and immediacy of the intervention. ${ }^{66}$ In addition, more efficient treatment time means faster acquisition and maintenance of skills. Utilizing tools such as SARs may prove to be a beneficial method for improving the capacity and efficacy of interventions all around. If such interventions are affordable, portable, and increase engagement, then they could be used in the schools and at home, providing an intervention tool that may increase therapy hours without increasing the cost. Begum et al claim that in order to realize all of the efforts in HRI and SARs, it is essential to prove the utility of robots in ASD therapy based on established guidelines to determine the strength of evidence from an experimental intervention so that it can be established as an evidence-based practice in autism therapy. ${ }^{10}$ Kim et al elaborate on this by noting that in order to shift toward studies on SARs that adhere to established clinical standards, there is a need for more standardized recruitment, reporting, and analysis with respect to control groups. ${ }^{39}$

As pointed out by several researchers, ${ }^{7,10,15,39}$ missing from the research in this field of HRI are the generalization of social skills from robot-human interactions, clinical effectiveness in conducting ABA on larger treatment samples, using published scales to assess social deficits and early diagnosis, as well as maintaining treatment integrity. Thus, SARs can be seen as a vehicle to reduce the barriers that keep children with ASD from receiving affordable and effective treatment. What is needed now, more than ever, is more collaboration between the engineers who develop SARs and behavior analysts. With both areas of expertise overlapping in the development of SARs for the benefit of those who we serve, a better future is possible for everyone involved.

\section{Acknowledgments}

This work is supported by internal funding from Salem State University and Worcester Polytechnic Institute.

\section{Disclosure}

The authors report no conflicts of interest in this work.

\section{References}

1. Ferrari E, Robins B, Dautenhahn K. Therapeutic and educational objectives in robot assisted play for children with autism. Paper presented at the 18th IEEE International Workshop on Robot and Human Interactive Communication - RO-MAN 2009; 2009; Toyama, Japan.

2. Barakova E, Lourens T, José Manuel Ferrández Vicente, José Ramón Álvarez Sánchez, Félix de la Paz López, Fco. Javier Toledo Moreo, editors. Interplay between natural and artificial intelligence in training autistic children with robots. In: Natural and Artificial Models in Computation and Biology. Heidelberg, Berlin: Springer-Verlag; 2013:161-170.

3. Cabibihan J-J, Javed H, Ang M Jr, Aljunied SM. Why robots? A survey on the roles and benefits of social robots in the therapy of children with autism. Int J Soc Robot. 2013;5(4):593-618.

4. Feil-Seifer D, Matarić M. Defining socially assistive robotics. In: Proceedings of the International Conference on Rehabilitation Robotics; June, 2005; Chicago, IL; 465-468.

5. Goldsmith TR, LeBlanc LA. Use of technology in interventions for children with autism. JEIBI. 2004;1(2):166.

6. Reed FDD, Hyman SR, Hirst JM. Applications of technology to teach social skills to children with autism. Res Autism Spectr Disord. 2011;5(3):1003-1010.

7. Grynszpan O, Weiss PL, Perez-Diaz F, Gal E. Innovative technologybased interventions for autism spectrum disorders: a meta-analysis. Autism. 2014;18(4):346-361.

8. Dickstein-Fischer L, Alexander E, Xiaoan Y, Hao S, Harrington K, Fischer GS. An affordable compact humanoid robot for autism spectrum disorder interventions in children. Poster presented at: The 33rd Annual International Conference of the IEEE EMBS; August, 2011; Boston, USA.

9. Scassellati B, Admoni H, Matarić, M. Robots for use in autism research. Annu Rev Biomed Eng. 2012;14:275-294.

10. Begum M, Serna RW, Yanco HA. Are robots ready to deliver autism interventions? A comprehensive review. Int J Soc Robot. 2016;8(2): 157-181.

11. Robins B, Dautenhahn K, Dubowski J. Does appearance matter in the interaction of children with autism with a humanoid robot? Interact Stud. 2006;7(3):509-542.

12. Scassellati B. How social robots will help us to diagnose, treat, and understand autism. In: Siciliano B, Khatib O, Groen F, editors, Springer Tracts in Advanced Robotics: Robotics Research, Berlin: Springer; 2007:552-563.

13. Robins B, Otero N, Ferrari E, Dautenhahn K. Eliciting requirements for a robotic toy for children with autism - results from user panels. Poster presented at: The 16th IEEE International Symposium on Robot and Human Interactive Communication - RO-MAN 2007; August, 2007; Jeju Island, Korea.

14. Ricks DJ, Colton MB. Trends and considerations in robot-assisted autism therapy. Poster presented at: The 2010 IEEE International Conference on Robotics and Automation (ICRA), May, 2010; Anchorage, AK. 
15. Diehl JJ, Schmitt LM, Villano M, Crowell CR. The clinical use of robots for individuals with autism spectrum disorders: a critical review. Res Autism Spectr Disord. 2012;6(1):249-262.

16. Center for Disease Control and Prevention (CDC). Prevalence of Autism Spectrum Disorder Among Children Aged 8 Years - Autism and Developmental Disabilities Monitoring Network, 11 Sites, United States, 2010. MMWR. Morbidity and Mortality Weekly Reports. 2014;63(2):1-21. Available from: https:/www.cdc.gov/mmwr/preview/ mmwrhtml/ss6302a1.htm. Accessed March 17, 2018.

17. American Psychiatric Association. Diagnostic and Statistical Manual of Mental Disorders. 5th ed. Washington, DC: American Psychiatric Association; 2013.

18. Fombonne E. Epidemiology of pervasive developmentaldisorders. Pediatr Res. 2009;65:591-598.

19. Skuse DH. Imprinting the $X$ chromosome, and the male brain: explaining sex differences in the liability to autism. Pediatr Res. 2000;47(1):9-16.

20. Jacquemont $\mathrm{S}$, Coe BP, Hersch $\mathrm{M}$, et al. A higher mutational burden in females supports a "Female Protective Model" in neurodevelopmental disorders. Am J Hum Genet. 2014;94(3):415-425.

21. Werling DM, Geschwind DH. Sex differences in autism spectrum disorders. Curr Opin Neurol. 2013;26(2):146-153.

22. Dawson M, Gernsbacher MA. Effectiveness of intensive autism programmes. Lancet. 2010;375(9716):722.

23. Wheeler JJ, Mayton MR, Carter SL. Methods for Teaching Students with Autism Spectrum Disorders: evidence-Based Practices. Pearson. 2014.

24. DeMyer MK, Hingtgen JN, Jackson RK. Infantile autism reviewed: a decade of research. Schizophr Bull. 1981;7(3):388-451.

25. Kasari C, Lawton K. New directions in behavioral treatment of autism spectrum disorders. Curr Opin Neurol. 2010;23(2):137-143.

26. Weitlauf AS, McPheeters ML, Peters B, et al. Therapies for Children with Autism Spectrum Disorder: Behavioral Interventions Update. Rockville, MD: US Department of Health and Human Services; 2014.

27. Baer DM, Wolf MM, Risley TR. Some current dimensions of applied behavior analysis. J Appl Behav Anal. 1968;1(1):91-97.

28. Baer DM, Wolf MM, Risley TR. Some still-current dimensions of applied behavior analysis. J Appl Behav Anal. 1987;20:313-327.

29. Buescher AVS, Cidav Z, Knapp M, Mandell DS. Costs of autism spectrum disorders in the united kingdom and the united states. JAMA Pediatrics. 2014;168(8):721-728.

30. Chasson GS, Harris GE, Neely WJ. Cost comparison of early intensive behavioral intervention and special education for children with autism. J Child Fam Stud. 2007;16(3):401-413.

31. Zane T, Davis C, Rosswurm M. The cost of fad treatments in autism. J Early Intensive Behav Interv. 2008;5(2):44-51.

32. Special Learning, Inc. Funding Overview. 2011. Avaiable from: https://www.special-learning.com/article/funding_overview. Accessed March 17, 2018.

33. Hastings RP, Symes MD. Early intensive behavioral intervention for children with autism: parental therapeutic self-efficacy. Res Dev Disabil. 2002;23(5):332-341.

34. Jennett HK, Harris SL, Mesibov GB. Commitment to philosophy, teacher efficacy, and burnout among teachers of children with autism. J Autism Dev Disord. 2003;33(6):583-593.

35. Gibson JA, Grey IM, Hastings RP. Supervisor support as a predictor of burnout and therapeutic self-efficacy in therapists working in ABA schools. J Autism Dev Disord. 2009;39(7):1024-1030.

36. LeBlanc LA, Gravina N, Carr JE. Training issues unique to autism spectrum disorders. In: Maston JL. editor, Applied Behavior Analysis for Children with Autism Spectrum Disorders. New York, NY: Springer Science+Business Media, LLC; 2009:225-235.

37. Grindle CF, Kovshoff H, Hastings RP, Remington B. Parents' experiences of home-based applied behavior analysis programs for young children with autism. J Autism Dev Disord. 2009;39(1):42-56.
38. Hurt AA, Grist CL, Malesky LA, McCord DM. Personality traits associated with occupational 'burnout' in ABA therapists. J Appl Res Intellect Disabil. 2013;26(4):299-308.

39. Kim E, Paul R, Shic F, Scassellati B. Bridging the research gap: making HRI useful to individuals with autism. JHRI. 2012;26-54.

40. Dautenhahn K, Werry I. Towards interactive robots in autism therapy: background, motivation and challenges. Pragmatics and Cognition. 2004;12(1):1-35.

41. Moon A. NAO Next Gen now available for a wider audience. 2014. Available from: http://robohub.org/nao-next-gen-now-availablefor-the-consumer-market/. Accessed March 17, 2018.

42. Robotlab. Engage! K12 transforms every class into a stem-class. 2016. Available from: http://www.robotlab.com. Accessed March 17, 2018.

43. Robots For Autism. (n.d.). About Milo. Available from: https:// robotsforautism.weebly.com/about-milo.html. Accessed March 17, 2018.

44. Welch KC, Lahiri U, Warren Z, Sarkar N. An approach to the design of socially acceptable robots for children with autism spectrum disorders. Int J Soc Robot. 2010;2(4):391-403.

45. Tariq S, Baber S, Ashfaq A, Ayaz Y, Naveed M, Mohsin S. Interactive therapy approach through collaborative physical play between a socially assistive humanoid robot and children with autism spectrum disorder. In: International Conference on Social Robotics; November, 2016:561-570; Kansas City, MO.

46. Giullian N, Ricks D, Atherton A, Colton M, Goodrich MA, Brinton B. Detailed requirements for robots in autism therapy. Poster presented at: The IEEE International Conference on Systems, Man and Cybernetics (SMC); October, 2010; Istanbul, Turkey.

47. Curtis A, Shim J, Gargas E, Srinivasan A, Howard AM. Dance dance Pleo: developing a low-cost learning robotic dance therapy aid. Poster presented at: The Proceedings of the 10th International Conference on Interaction Design and Children; June, 2011; Ann Arbor, Michigan.

48. Kozima H, Michalowski MP, Nakagawa C. Keepon: a playful robot for research, therapy, and entertainment. Int J Soc Robot. 2009; $1(1): 3-18$

49. Shibata T, Kawaguchi Y, Wada K. Investigation on people living with seal robot at home: analysis of owners' gender differences and pet ownership experience. Int J Soc Robot. 2011;4(1):53-63.

50. Jentsch E. Zur Psychologie des Unheimlichen. Psychiatrisch-Neurologische Wochenschrift. 1906;8(22):195-198.

51. Mori M. The uncanny valley. Energy. 1970;7(4):33-35.

52. Publications - Ask Nao. 2017. Available from: https://asknao.aldebaran. com/publications. Accessed March 17, 2018.

53. Salvador M, Marsh AS, Gutierrez A, Mahoor MH. Development of an ABA autism intervention delivered by a humanoid robot. In: International Conference on Social Robotics; November 2016; 551-560; Kansas City, MO.

54. Skinner BF. Teaching machines: from the experimental study of learning come devices which arrange optimal conditions for self-instruction. Science. 1958;128(3330):969-977

55. Keller F. Good-bye teacher. J Appl Behav Anal. 1968:1(1):79-89.

56. Pear JJ, Crone-Todd DE. Personalized system of instruction in cyberspace. J Appl Behav Anal. 1999;32(2):205-209. doi:10.1901/ jaba.1999.32-205

57. Ortega JV, Iwata BA, Nogales-González C, Frades B. Assessment of preference for edible and leisure items in individuals with dementia. J Appl Behav Anal. 2012;45(4):839-844.

58. Laraway S, Snycerski S, Michael J, Poling A. Motivating operations and terms to describe them: some further refinements. J Appl Behav Anal. 2003;36(3):407-414

59. Michael J. Distinguishing between discriminative and motivational functions of stimuli. J Exp Anal Behav. 1982;37(1):149-155.

60. Shogren KA, Faggella-Luby MN, Bae SJ, Wehmeyer ML. The effect of choice-making as an intervention for problem behavior a meta-analysis. J Posit Behav Interv. 2004;6(4):228-237.

61. Dautenhahn K. Roles and functions of robots in human society: implications from research in autism therapy. Robotica. 2003;21(04):443-452. 
62. Dickstein-Fischer L, Fischer GS. Combining psychological and engineering approaches to utilizing social robots with children with Autism. Poster presented at: The 36th Annual International Conference of the IEEE Engineering in Medicine and Biology Society (EMBC); August, 2014; Chicago, IL.

63. Dickstein-Fischer LA, Pereira RH, Gandomi KY, Fathima AT, Fischer GS. Interactive tracking for robot-assisted autism therapy. In: Proceedings of the Companion of the $2017 \mathrm{ACM} / \mathrm{IEEE}$ International Conference on Human-Robot Interaction; March 2017; 107-108; Vienna, Austria.
64. Corsello CM. Early intervention in autism. Infants and Young Children 2005;18(2):74-85.

65. Pipkin CSP, Vollmer TR, Sloman KN. Effects of treatment integrity failures during differential reinforcement of alternative behavior: a translational model. J Appl Behav Anal. 2010;43(1):47-70.

66. Symes MD, Remington B, Brown T, Hastings RP. Early intensive behavioral intervention for children with autism: therapists' perspectives on achieving procedural fidelity. Res Dev Disabil. 2006;27(1):30-42.
Innovation and Entrepreneurship in Health

\section{Publish your work in this journal}

Innovation and Entrepreneurship in Health is an international, peer reviewed, open access journal publishing original research, reports, reviews and commentaries on innovation and entrepreneurship in health. Special focus will be given to the theory, process, and practice of innovation and entrepreneurship by individuals and organizations

\section{Dovepress}

within the health care context globally. The manuscript management system is completely online and includes a very quick and fair peer review system, which is all easy to use. Visit http://www.dovepress.com/ testimonials.php to read real quotes from published authors.

Submit your manuscript here: https://www.dovepress.com/innovation-and-entrepreneurship-in-health-journal 